\title{
Strain imaging of internal deformation
}

\author{
Jerome J. Mai and Michael F. Insana \\ Department of Biomedical Engineering \\ University of California at Davis
}

Corresponding Author: Michael Insana, Department of Biomedical Engineering

University of California, One Shields Avenue, Davis, CA 95616, USA

Phone (530) 752-0288

Fax (530) 754-5739

mfinsana@ucdavis.edu 


\begin{abstract}
A tissue-like gelatin elasticity-flow phantom was examined to develop ultrasonic strain imaging for the detection of internal pulsatile deformations. The same imaging technique was then applied in vivo to monitor deformation in tissues surrounding the normal brachial artery. The results suggest that vascular strain patterns resulting from biological stimuli are very different from those generated using externally applied stress fields, and are directly related to pressure variations within the vessel. These data suggest a potential role for strain imaging in measuring local vascular pressure or compliance non-invasively.
\end{abstract}

Keywords: brachial artery, elasticity, phantoms, pulsatile flow, strain imaging, ultrasound, vascular imaging 


\section{INTRODUCTION}

Several techniques use signals from imaging systems to form diagnostic images that describe the spatial and temporal distributions of tissue elasticity, in vivo. Ultrasonic applications alone span a broad range from tumor detection (Garra et al. 1997) to characterization of vascular plaques (de Korte et al. 2000) to the study of skeletal muscle contraction (Levinson et al. 1995) to the assessment of fetal lung maturity (Adler et al. 1990). Today, the most common approaches measure soft tissue strain produced by an external stress stimulus, either statically applied by the imaging transducer or a vascular balloon, or dynamically applied by introducing shear waves into the body. However the study of ultrasonic elasticity began with Dickinson and Hill (1982), Wilson and Robinson (1982), and Tristam et al. (1986) who measured deformations of tissues, including tumors, from natural internal stress stimuli such as cardiac contractions. These are dynamic analyses that have been limited to single A-line or M-mode analyses of tissue because of the technology available at the time. Each describes tissue kinetics but with little or no spatial resolution.

The technology exists today for recording the complex envelope of echo samples for extended fields of view at video frame rates using laboratory and commercial systems. Thus it is now possible to apply wide-band signal processing analysis to measure displacements along the beam axis as small as $5^{\circ}$ of phase $(3 \mu \mathrm{m}$ at $7 \mathrm{MHz}$ ) or with a spatial resolution comparable to B-mode imaging (Insana et al. 2000). We are exploring this opportunity to image blood vessels, specifically, to visualize viscoelastic

deformations surrounding vessels during cardiac pulse propagation. Potential applications of this approach include local measurements of arterial pressure or vascular compliance, plaque 
characterization, and the development of noninvasive methods for studying the relationship between blood-flow dynamics and tissue elasticity.

This report describes our initial investigations using a tissue-like elasticity-flow phantom with flow channels to which we applied static or pulsatile hydraulic pressures. The intent is to assess the sensitivity of displacement and strain measurements in a relatively simple tissue-like media. Finally, in vivo measurements in a brachial artery of a normal volunteer are presented.

\section{MATERIALS AND METHODS}

\subsection{Materials}

A well-known hydro-gel material formed the bulk of the ultrasonic elasticity-flow phantom constructed for this study (Hall et al. 1997; Zagzebski and Madsen 1995). The material composition by mass was 83.93\% distilled water, 7.72\% n-propanol, 5.04\% animal-hide gelatin powder (275 bloom), 0.05\% formaldehyde, and 3.26\% graphite powder. These ingredients provided a durable viscoelastic material with temporally stable acoustic and elastic properties in the range of many soft tissues. The molten mixture was poured into a $10 \times 8 \times 7.4 \mathrm{~cm}^{3}$ acrylic container and slowly rotated while congealing to keep the graphite particles suspended. Nine 1.3-mm-diameter acrylic rods were positioned centrally in the rectangular container, each parallel to the others and separated $10 \mathrm{~mm}$ on center. They were removed after the gel congealed to provide wall-less fluid channels in the gel block (Fig 1). Previous phantom designs include rigid flow channels inappropriate for this study (Rickey et al. 1995) or elastic walls that are made of highly attenuating material. Stiff plastic tubes were securely inserted $5 \mathrm{~mm}$ into channel as inlets and outlets to connect the phantom to flow sources and sinks. Fluid flowed through 
channels under gravitational feed for steady flow and under peristaltic pump feed for pulsed flow. The fluid was a weak suspension of cornstarch in water to provide some ultrasonic scattering. The acoustic properties of the fluid were not measured.

\subsection{Acoustic measurements}

One extra sample of the graphite-gelatin material of 2.54-cm thickness was prepared during phantom construction for measurements of acoustic properties. Sound speed and attenuation coefficient were measured at $21^{\circ} \mathrm{C}$ using the narrow-band, through-transmission substitution technique of Madsen et al. (1982). The attenuation coefficient at $5 \mathrm{MHz}$ was $1.30 \mathrm{~dB} / \mathrm{cm}$ and the speed of sound was $1561 \mathrm{~m} / \mathrm{s}$. Attenuation in this material is known to increase approximately linearly with frequency (Madsen 1978). The attenuation measurement was calibrated using a castor oil sample (Madsen 1982). The measured value $12.10 \mathrm{~dB} / \mathrm{cm}$ and predicted value $11.85 \mathrm{~dB} / \mathrm{cm}$ for attenuation agreed within $10 \%$.

\subsection{Viscoelastic measurements}

We also measured the elastic modulus of the graphite-gelatin material using an indentation test on the surface of the elasticity-flow phantom (Kargel 2001). A computer-controlled linear positioner, with 0.1$\mathrm{mm}$ positional precision, and a flat-ended cylindrical indentor (radius $a=2.5 \mathrm{~mm}$ ) provided the compression to the sample. A digital electronic balance with negligible movement measured the load with a precision of $0.01 \mathrm{~g}$. The apparatus was controlled under Labview to record force $(F)$ and indentation depth $(w)$ in real time. Measurements were obtained from least squares estimates of the data during the compressional phase for indentations between 0.0 and $0.25 \mathrm{~mm}$, which is the range of the imaging experiments described below. Elastic modulus was calculated using the formula $E=F(1-$ 
$\left.v^{2}\right) / 2 a w \kappa$, assuming Poisson's ratio $v=0.498$ for nearly incompressible material and $\kappa=1$ for large sample thickness (Zhang et al. 1997). The elastic modulus estimate was $10.7 \mathrm{kPa}$.

\subsection{Ultrasonic echo recording}

To acquire echo data, we used a Siemens Elegra system with a 7.5 MHz linear array (7.5L40) transmitting 7.2 $\mathrm{MHz}$ broadband pulses. A single transmit focal zone was centered on the target. Viewing the real-time B-mode image display and adjusting the depth-gain compensation manually to give constant echo amplitude with depth over the region of interest, we acquired digitized echo signals from system memory. Echo frames containing 312 data segments $(0.12 \mathrm{~mm}$ transducer pitch) of inphase/quadrature (I/Q) time series were recorded at 15 frames per second (fps). A total of seventy-three echo frames were recorded from a region $51.3 \mathrm{~mm}$ deep and $38 \mathrm{~mm}$ wide. Radio-frequency (RF) signals were digitized at 36 Msamples/s, mixed to form I/Q data, downsampled by 2.5 for electronic transfer, and then upsampled by 4 before processing. We formed strain images from echo data with an effective (fast-time) RF data density of $57.6 \mathrm{Msamples} / \mathrm{s}$ and a bandwidth of $18 \mathrm{MHz}$. The 15-fps (slow-time) sampling rate provided about four cycles of pulsed flow data at 60 fluid pulse cycles $/ \mathrm{min}$. I/Q data were transferred via a network connection for offline processing on a Pentium III computer.

\subsection{Strain image formation}

Strain images are formed from correlation-based displacement estimates. Local displacements are determined from pairs of RF data frames acquired for the same region in the object but at two times representing different stages of deformation. The Chaturvedi algorithm (1998a, 1998b) was used for all displacement estimates and strain images reported in this study. The algorithm was originally developed for imaging strain resulting from external compression, but is appropriate for imaging internal 
deformation from pulsed flow since displacements are usually small. From segmentation of the channel margins detected in B-mode images reconstructed from the high-density I/Q data, we were able to quantitatively determine that the maximum radial displacement for pulsatile flow measured along the sound beam approximately $0.1 \mathrm{~mm}$.

With this algorithm, we compute local displacements within the scan plane of the deformed object from 2-D correlation lags determined at three spatial scales. First, the coarsest estimates of displacement are used to measure and compensate for the overall (average) displacement and strain applied to one frame with respect to another. The process of warping echo data in a frame to compensate for the average physical deformation is known as global companding. Second, displacements measured at an intermediate-size spatial resolution (the 2-D data kernel was 1 x $1 \mathrm{~mm}^{2}$ ) are recorded and used to warp the echo fields via a local companding process. Finally, displacements are measured at the highest spatial resolution by 1-D correlation of twice companded echo frames. Displacement measurements from one stage of estimation improve the condition of the data for estimation at higher resolution (Insana et al. 2000). Companding is a 2-D analogue of the 1-D "temporal stretching" technique implemented by others (Cespedes and Ophir 1993; Cespedes et al. 1993; Varghese et al. 1996a, 1996b). Finally, components of displacement along the ultrasonic beam axis at each stage are summed and filtered by a two-sample FIR differentiator to form strain images. Axial strain pixel size is determined by parameters set at the final stage of displacement estimations. However, each stage of the estimation process and the echo impulse response of the instrumentation determine spatial resolution of the strain image. 
For internal deformation imaging, global companding is not used for phantoms but is applied when scanning in vivo. Although the average strain expected in an echo frame recorded during pulsed flow is zero, global companding compensates for in-plane transducer motion at the skin surface that commonly occurs during live scanning.

\section{RESULTS}

\subsection{Phantom imaging with external stimulus}

The elasticity-flow phantom was mounted in an acrylic fixture, placed in distilled water, and scanned along a central plane with a linear array transducer (Fig 1). In this phase of the study, and contrary to that shown in Fig 1, channels were not connected to pressure sources (water column or pump) and boundaries were confined only to prevent out-of-plane motion; the phantom was free to move within the scan plane. The pre-compression B-mode image and corresponding strain image with $2 \%$ applied compressive strain are shown in Figs $2 a$ and $2 b$, respectively. Channels were filled with distilled water that moved freely when the phantom was deformed. As expected, strains around the channels are larger than in the background (bright pixels in Fig. $2 b$ indicate soft areas). By extending the length of the correlation window to minimize strain noise, we introduced shape distortions: channels appear much larger in the vertical dimension than their actual 1.3-mm-diameter size. Also, decorrelation errors are observed above and below each channel, particularly in the bottom row where defocusing reduces the echo signal-to-noise ratio. Decorrelation errors (high-contrast black and white pixels) are seen where strain gradients are large or shear strains are significant, which is often the case near region boundaries with sharp elastic modulus contrast. Decorrelation errors can be mitigated in some situations by adjusting the correlation window size, as described in the Discussion section below. 


\subsection{Phantom imaging with internal stimulus}

The apparatus illustrated in Fig 1 was used for imaging local deformation from static or pulsatile pressures applied to the top-center flow channel. In this phase of the study, no external stress was applied and each exterior surface was rigidly confined in the acrylic mold. In the first experiment, hydrodynamic pressures were produced with a peristaltic pump (Gilson Minipuls 2) that generated pulsatile flow within a closed loop in the top-center channel. The pressure pulse rate was roughly 1 pulse/s with a time average flow of $0.114 \mathrm{ml} / \mathrm{s}$. In the second experiment, hydrostatic pressures were generated in the same channel by connecting one side to a water column and blocking flow in the other. In either case, the image plane was located near the center of the phantom, perpendicular to the channel axis. Tygon tubing that was much stiffer than the phantom material joined the pump or water column to the phantom channel. Consequently, nearly all of the deformation from the pressure pulse occurred inside the phantom channel. The pressure variation inside the channel was also measured at the time of image acquisition with a medical pressure transducer system (Medex LogiCal pressure transducer, Puritan-Bennett Co. PB240 patient monitor).

\subsubsection{Pulsatile pressure}

Deformation varies with time for pulsatile flow. Therefore one of the echo frames must be selected as a reference to compute the sequence of strain images. The choice of reference frame is arbitrary yet it dramatically influences strain contrast. To simplify interpretation of the strain patterns, we chose the reference frame at the time when the channel pressure was lowest (smallest channel diameter) out of the 16 frames recorded for one complete cycle. Consequently, by definition, all movement in the scan plane was directed radially outward from the channel, and the time-varying strains were compressive. 
Fig 2c shows a typical strain image of the phantom with the top-center channel under internal stimulus. Though the same region of interest is displayed, strain patterns in Fig 2c are very different from those of the external stimulus in Fig 2b. First, the local nature of the stress field means that only deformed structures near the channel appear in the image. Second, the deformation pattern is distorted vertically because we selected a long, narrow correlation window to reduce the strain noise caused by echo noise. Third, some regions appear bright, indicating compressive strain, while others appear dark, indicating an apparent tensile strain. Although we know that the strains are compressive and radially directed, the linear array transducer only senses displacements along the axis of the sound beam. Strain patterns in Figs $2 \mathrm{~b}$ and $2 \mathrm{c}$ are both determined by the elastic modulus of the medium and the applied stress field. Unlike that in Fig 2b, the stress field in the images of Fig 2c is time varying, acts locally, and is radially directed. Strain values in Fig 2c exhibit a dipolar spatial pattern because the measurements depend strongly on the orientation of the sound beam with respect to the movement. The appearance of strain patterns seen in Fig 2c is explained in terms of the beam geometry in the Discussion section.

Figs 3a-c, separated by $1 / 3 \mathrm{sec}$ between each image, show the strain images at three different stages in one pulse cycle, all displayed in the same region of interest as the zoomed-in B-mode image in Fig 3d. Fig $3 a$ is near the start of the pulse when the channel was least expanded, so most of the image is gray (zero strain). As the channel pressure increases, the expanded channel deforms the surrounding medium, thus providing strain contrast. In Fig 3c, which was near the peak of the pulse cycle, decorrelation effects can be seen around the channel. Both Fig $3 b$ and $3 \mathrm{e}$ were generated with the same echo frame pair, but $3 \mathrm{~b}$ was processed with a correlation window of $3.33 \mathrm{~mm}$, while $3 \mathrm{e}$ was done with $0.42 \mathrm{~mm}$. Though the strain patterns in Fig 3e give a more realistic description of the channel, the 
decorrelation noise has significantly increased. Also the aforementioned dipolar pattern becomes more obvious in Fig 3e (arrow).

To study how the internal stimulus affects the strain images, we took advantage of the simple phantom geometry to find a relationship between channel pressure and local phantom strain. We were able to adopt a 2-D elasticity model (Volterra and Gaines 1971) that predicts displacements in a wallless channel along lines radial from the center of the channel $u_{r}(r, t)$ at radial distance $r$, time $t$, and at channel pressure $P(t)$ :

$$
u_{r}(r, t)=\frac{(1+v) D^{2}}{4 r E} P(t), \quad \text { for } r \geq D / 2 .
$$

$E$ is the elastic modulus of the gelatin material surround the channel, $v$ is the Poisson ratio of the gelatin, and $D$ is the reference frame channel diameter. The corresponding radial strain in the object is

$$
\varepsilon_{r}(r, t)=\left|\frac{d u_{r}(r, t)}{d r}\right|=\frac{(1+v) D^{2}}{4 r^{2} E} P(t) .
$$

As in conventional strain imaging, compressive strain is defined as a positive quantity. Eqs. (1) and (2) predict that radial displacements and strains are proportional to pressure inside the channel. To test this prediction, we first plotted the pressure profile within one cycle inside the channel by averaging over several cycles (Fig 4a). We then calculated for one pulse cycle the averaged strain magnitude in a rectangular region $\left(0.7 \times 0.8 \mathrm{~mm}^{2}\right.$, boxed region in Fig $\left.3 \mathrm{~b}\right)$ that is $1.8 \mathrm{~mm}$ to the right of the center of the, as shown in Fig 4b. This region, processed with a 3.33-mm correlation window, was picked for its low level of decorrelation. Though a phase lag might exist between the two plots in Fig 4, we can easily see the similarity between them and that the strain is directly related to the amount of pressure inside the channel. The maximum strain in the pulse cycle was reached with a pressure of $6.8-\mathrm{mm} \mathrm{Hg}$. 


\subsubsection{Hydrostatic pressure}

A strain-pressure calibration curve was also measured for the hydrostatic pressure inside the phantom generated by the water column. Under controlled static pressure, the relationship between pressure and strain can be studied without being affected by variables like flow and the phase lag in pulse propagation. The "zero pressure" water height was established by letting water move freely through the unsealed ends of the channel when the phantom was completely submerged in water. The echo frame acquired at this "zero pressure" was used as reference frame for generating strain images. After sealing off the outlet of the channel, water was then added to the column at $1-\mathrm{cm}$ increments to a maximum height of $15 \mathrm{~cm}$, which converts to a pressure of $11.07-\mathrm{mm} \mathrm{Hg}$. Since the reading of the water height was more error-prone, the real pressure variation was also monitored with a pressure transducer and averaged over five seconds at each step (Fig 5a). Echo data were taken and a strain image was processed at each pressure increment. The averaged strain magnitude in the aforementioned region (boxed region in Fig 5b) was calculated as well for the 16 pressure levels. The averaged strain magnitude (Fig 5c) increased linearly with pressure between 1-11 mm Hg. Comparing Figs 4 and 5, we see that the proportionality between pressure and strain holds for both static and dynamically stresses.

\subsection{Brachial artery imaging in vivo}

Brachial artery was chosen for in vivo testing because systemic blood pressure is conventionally measured there on the upper arm with a sphygmomanometer. Also, this location is acoustically accessible and provides reasonable echo signal strength and imaging resolution. The orientation of the arm and the location of the artery can make full acoustic contact difficult. We obtained reproducible sets of echo data by mounting the transducer at an oblique angle with a rotary device and a linear positioner (Fig. 6). The arm rested on the transducer surface that is flush mounted to a flat plate. 
Abundant coupling gel and the weight of the arm pressing the skin against the transducer ensured full acoustic contact. The elbow is raised slightly above shoulder height to ensure no pooling of blood in the veins in the nearby region. Subjects held their breath during data acquisition to minimize external movement. We acquired echo data from several consecutive cardiac cycles at $15 \mathrm{fps}$. The frame with the smallest arterial diameter, corresponding to end diastole, is the reference frame for generating strain images. The data recorded of the right arm of a healthy 25 -year-old male volunteer with a blood pressure reading of 135/80 $\mathrm{mm} \mathrm{Hg}$ are displayed in this study.

Fig. 7a shows a B-mode image of the upper arm region containing the brachial artery at end diastole. The brachial artery is the dark circular object in the mid-right region of the B-mode image; it is approximately $3 \mathrm{~mm}$ in diameter at end diastole. Three muscle groups separated by fascia surround this artery: bicep, brachialis, and triceps. Smaller arteries, veins, and nerves covered by fascia also reside in the regions next to the brachial artery. We generated 46 strain images over three cardiac cycles. Fig 7be are four strain images from peak systole to near end diastole separated in time by $1 / 4 \mathrm{sec}$ and generated with a $3.33-\mathrm{mm}$-long correlation window. The artery is most distended at peak systole and then gradually returns to minimal size. Vascular pulsatility in Fig 7 produces strain patterns similar to those of the pulsing phantom channel in Fig 3, although it is clear that the heterogeneity of surrounding tissues in the arm have a strong affect on strain images. For example, the humerus bone, which appears in the lower left corner of the B-mode image, seems to act as a rigid boundary that limits deformation of the tissue surround the vessels. The dipolar strain pattern is apparent above the vessels but not below. Other artifacts also exist in these images. Three smaller internal deformation strain patterns, which may be caused by smaller vessels, are also visible to the left of the brachial artery. These effects are much clearer with short correlation windows $(0.83 \mathrm{~mm}$, Fig $7 \mathrm{f})$, though the compromise between spatial 
resolution and noise that occurs by varying the window length is apparent. Strain imaging appears capable of detecting deformation asymmetries such as those caused by the proximity of the humerus bone, and its high sensitivity to small strain variations suggests the possibility of detecting regional arterial compliance changes caused by the formation of plaques and calcification.

We measured one mean regional strain magnitude in a $0.6 \times 1.0 \mathrm{~mm}^{2}$ region (boxed region in Fig 7d) at the brachial artery for each strain image in our time series. These values are plotted versus time for three cardiac cycles in Fig. 8a. Though the smoothness of this plot is limited by the frame rate at which echo data were collected, the plot clearly depicts the essential characteristics of brachial arterial pressure profile seen in Fig. 8b (from Remington and Wood 1956; Salans et al. 1951). Comparing these curves, we observe that there is a close relationship between the variations in cardiac pressure and those from the resultant strain. If we increase the pressure of the transducer against the arm, we find larger fluctuations in strain surrounding the brachial artery during each cardiac cycle. This response is expected - external force increases vascular resistance in the measurement region by adding pressure in the surrounding tissue to reduce the vessel cross sectional area. It is important to maintain a consistent and minimal pressure between the transducer and skin surfaces to avoid disturbing the natural deformation of tissues surrounding arteries during the cardiac cycle.

\section{DISCUSSION}

\subsection{Interpretation of strain patterns from pulsatile pressure}

Strain patterns near a pulsing vessel are very different from those formed with external compression. The pressure pulse in the channel expands the walls radially outward (Fig 9a) thus compressing the 
surrounding regions. Pulse-echo ultrasound can only sense these very tiny movements if they occur along the direction of wave propagation (Fig 9b). Consequently the echo data records only a component of the displacement vector, that in the direction of the beam axis, $z . u_{z}(r, t)$ is proportional to $u_{r}(r, t)$. For uniform radial displacement, we can assume

$$
u_{z}(r, \theta, t)=u_{r}(r, t) \cos \theta, \quad \text { for } r \geq D / 2 .
$$

The angle $\theta$ defines the direction of motion in the imaging plane with respect to $z$ (see Eq 1). As shown in Fig 9b, the axial strain, i.e., the strain component along the beam axis, is compressive (bright pixels) in regions above and below the vessel, near $\theta=0, \pi$. However, the axial strain occurring in regions lateral to the vessel, near $\theta= \pm \pi / 2$, is tensile (dark pixels). The radial projection of displacement onto the $z$-axis produces a dipole pattern in the strain image. The dipole pattern is nearly symmetric in the phantom because radial expansion is almost uniform over $\theta$. The weight of the phantom itself might have caused parts of the phantom to be more compressed than other parts so the strain patterns are not perfectly symmetric. Images of the brachial artery, however, show that heterogeneity of the elastic medium surrounding the vessel produces an asymmetric strain pattern. Notice that if the frame with the most expanded channel were used as the reference for computing the strain image sequence, the grayscale polarity of the strain image would reverse such that bright regions would become dark and dark regions bright. If a frame anywhere else during the pulse cycle were used as the reference, though the amount of decorrelation may be lowered, the polarity of the strain patterns would shift during the pulse cycle.

The phantom and brachial artery strain images suggest that regions with pulsatile blood flow can be mistaken for hard or soft targets when imaging with external compression. However, the strain patterns are characteristically different, which aids discrimination. Similarly Bilgen (1998) found that 
strain patterns surrounding targets with circular cross sectional areas reveal information about the outof-plane dimension for imaging with external stimuli.

\subsection{Spatio-temporal strain variations across an expanding fluid channel}

Spatially, the largest displacement occurs at the channel wall, at $r=D / 2$ in Eq 1, and is reduced in proportion to the distance from the wall. We illustrate in Fig 10 the displacement and strain that is measured using echo cross correlation along the beam axis and through the center of the channel. The left column explains the effects when the expansion is small or the correlation window is large relative to the diameter of the channel. The right column explains the effects for larger expansions and smaller correlation window lengths. Recall that strain is determined from displacements estimated over a correlation window. Pixels in a strain image correspond to movement of the correlation window to different locations.

When the correlation window, represented by the line segment "I" is placed outside the channel (region $\boldsymbol{I}$ at top left in Fig 10a), we find the displacement averaged over the echo segment. Displacement in homogeneous viscoelastic media varies smoothly over space, so echo decorrelation will be minimal as long as the strain over the correlation window dimension is small, less than a one percent. As the correlation window enters the low scattering fluid channel (region $\boldsymbol{I I}$ in Fig 10a), displacement estimates are dominated by echoes at the channel wall, so the displacement plateaus at a peak. When the correlation window spans the channel to include echoes from both sides (region III in Fig 10a), the average displacement falls rapidly to zero if the channel expands symmetrically. When the correlation window is positioned below the channel, as in regions $\boldsymbol{I} \boldsymbol{V}$ and $\boldsymbol{V}$ in Fig 10a, we measure the same displacement profile except in the opposite direction. Computing the spatial derivative we find the 
strain. The simulated strain pattern in Fig 10a suggests there should be a bright and dark region centered at both walls of the channel. This is precisely what we observed in the images of Fig 3. We plot one line of displacement and strain measurements from the phantom data of Fig 3a in Figs $10 \mathrm{c}$ and e to show it is very similar to that predicted in Fig 10a.

If the extent of the channel expansion is large or the correlation window is small, then we observe the responses illustrated in Fig 10b. This is essentially a blurred version of the effects seen in Fig 10a. Regions $\boldsymbol{I I}$ and $\boldsymbol{I V}$ disappear due to the rapid drop in correlation between the reference frame and the displaced frame as the window shifts. Plotting a line of displacement and strain data taken from the image in Fig 3c and plotting them in Figs. 10d and f, we again see that measured patterns are similar to those predicted. In summary, the dynamic strain patterns from our simple tissue-like pulsed-flow phantom are easily interpreted in terms of the interaction between object motion the imaging system properties, and the estimation procedure.

\subsection{Relationships among vascular pressure, compliance and strain}

Studies have shown that vascular diameter varies proportionally with arterial pressure (Summa 1978). The phantom data presented in this report suggests that local strain is also proportional to the pressure inside the channel. However, the constant of proportionality depends on the stiffness of the vessel and materials surrounding the vessel. Investigators have chosen numerous ways to express the relationship among these three quantities (Lehmann 1999). One such expression is pressure-strain elastic modulus $E_{p}=\Delta P / \varepsilon_{r}$, where the strain is approximated as the relative change in vessel diameter $\varepsilon_{r} \cong \Delta D / D_{0}$ that occurs in response to the change in pressure (Peterson 1960). If $E_{p}$ is unknown but is constant over the physiological range of pressure variations $\Delta P$, then the strain 
measured near the vessel wall can be used to estimate the local pressure variation. This is what we observed from the data in Figs 4 and 5. Measuring peak systolic and end-diastolic pressures with the sphygmomanometer while imaging the brachial artery may enable us to relate strain to pressure as in Figs 4 and 5. These data should be acquired synchronously to detect any phase lag between the pressure and deformation pulses, which is likely because of the viscous response of blood vessels. Measurement of vascular compliance is the subject of a future study.

\section{CONCLUSIONS}

Time sequences of strain images are very sensitive to internal deformations such as those caused by pulsatile arterial flow, as shown by a simple phantom study using a clinical imaging system. Displacements of order $10 \mu \mathrm{m}$ and strains as small as 0.01 were quickly estimated in regions of area 0.7 $\mathrm{x} 0.8 \mathrm{~mm}^{2}$ about a $1.3-\mathrm{mm}$-diameter flow channel. The strain patterns are very different from those generated using external stimuli but are easily interpreted for the simple phantom geometry. These data suggest a potential role for real-time strain imaging in measuring local vascular pressure or compliance non-invasively.

Acknowledgements -- The authors gratefully acknowledge the contributions of William Hornof, Christian Kargel, and Dustin Kruse. This work was supported in part by NIH R01 CA 82497. 


\section{REFERENCES}

Adler RS, Rubin JM, Bland PH, Carson PL. Quantitative tissue motion analysis of digitized M-mode images: gestational differences of fetal lung. Ultrasound Med Biol 1990; 16:561-569.

Bilgen M, Insana MF. Elastostatics of a spherical inclusion in homogeneous biological media. Phys Med Biol 1998;43:1-20.

Cespedes I, Ophir J. Reduction of image noise in elastography. Ultrasonic Imaging 1993;15:89-102.

Cespedes I, Ophir J, Ponnekanti H, Yazdi Y, Li X. Elastography: elasticity imaging using ultrasound with application to muscle and breast in vivo. Ultrasonic Imaging 1993;15:73-88.

Chaturvedi P, Insana MF, Hall TJ. 2-D companding for noise reduction in strain imaging. IEEE Trans Ultrason Ferroelect Freq Contr 1998a;45:179-191.

Chaturvedi P, Insana MF, Hall TJ. Testing the limitations of 2-D companding for strain imaging using phantoms. IEEE Trans Ultrason Ferroelect Freq Contr 1998b;45:1022-1031.

de Korte CL, van der Steen AFW, Cespedes EI, Pasterkamp G, Carlier SG, Mastik F, Schoneveld AH, Serruys PW, Bom N. Characterization of plaque components and vulnerability with intravascular ultrasound elastography. Phys Med Biol 2000;45:1465-1475.

Dickinson RJ, Hill CR. Measurement of soft tissue motion using correlation between A-scans. Ultrasound Med Biol 1982;8:263-271.

Garra BS, Cespedes EI, Ophir J, Spratt SR, Zuurbier RA, Magnant CM, Pennanen MF. Elastography of breast lesions: initial clinical results. Radiology 1997;202:79-86.

Hall TJ, Bilgen M, Insana MF, Krouskop TA. Phantom materials for elastography. IEEE Trans Ultrason Ferroelect Freq Contr 1997;44:1355-1365.

Insana MF, Cook LT, Bilgen M, Chaturvedi P, Zhu Y. Maximum-likelihood approach to strain imaging using ultrasound. J Acoust Soc Am 2000;107:1421-1434. 
Kargel Ch, Trummer B, Plevnik G, Pellot-Barakat C, Mai JJ, Insana MF. Is Ultrasonic imaging a sensitive indicator of spatially varying elastic anisotropy? Proc IEEE Ultrasonics Symposium, Atlanta, October, 2001.

Lehmann ED. Terminology for the definition of arterial elastic properties. Path Biol 1999;47:656-664.

Levinson SF, Shinagawa M, Sato T. Sonoelastic determination of human skeletal muscle elasticity. J Biomech 1995;28:1145-1154.

Madsen EL, Zagzebski JA, Banjavic RA, Jutila RE. Tissue mimicking materials for ultrasound phantoms. Med Phys 1978;5:391-394.

Madsen EL, Zagzebski JA, Frank GR, Oil-in-gelatin dispersions for use in ultrasonically tissuemimicking materials. Ultrason Med Biol 1982;8:277-287.

Peterson LH, Jensen RE, Parnell J. Mechanical properties of arteries in vivo. Circ Res 1960;8:622-639.

Remington JW, Wood EH. Formation of peripheral pulse contour in man. J App Physiol 1956;9:433442.

Rickey DW, Picot PA, Christopher DA, Fenster A. A wall-less vessel phantom for Doppler ultrasound studies. Ultrasound Med Biol 1995;21:1163-1176.

Salans AH, Katz LN, Graham GR, et al. A study of the central and peripheral arterial pressure pulse in man: correlation with simultaneously recorded electrokymograms. Circulation 1951;4:510-521.

Summa Y. Determination of the tangential elastic modulus of human arteries in vivo. In: Bauer RD, Busse R, ed. The arterial system: dynamics, control theory and regulation. Berlin; Heidelberg; New York: Springer-Verlag, 1978; 95-100.

Tristam M, Barbosa DC, Cosgrove DO, Nassiri DK, Bamber JC, Hill CR. Ultrasonic study of in vivo kinetic characteristics of human tissue. Ultrasound Med Biol 1986; 12:927-937. 
Varghese T, Ophir J. Performance optimization in elastography: multicompression with temporal stretching. Ultrasonic Imaging 1996a;18:193-214.

Varghese T, Ophir J, Cespedes I. Noise reduction in elastograms using temporal stretching with multicompression averaging. Ultrasound Med Biol 1996b;22:1043-1052.

Volterra E, Gaines JH. Advanced strength of materials. New Jersey, Prentice-Hall, 1971.

Wilson LS, Robinson DE. Ultrasonic measurement of small displacements and deformations of tissue. Ultrason Imaging 1982; 4:71-82.

Zagzebski JA, Madsen EL. Ultrasound phantoms - concepts and construction. In: Goldman LW, Fowlkes JB, ed. Medical CT \& ultrasound: current technology and applications. Madison WI: Advanced Medical Publishing, 1995:121-142.

Zhang M, Zheng YP. Estimating the effective Young's modulus of soft tissues from indentation tests nonlinear finite element analysis of effects of friction and large deformation. Med Eng Phys 1997; 19:512-517. 


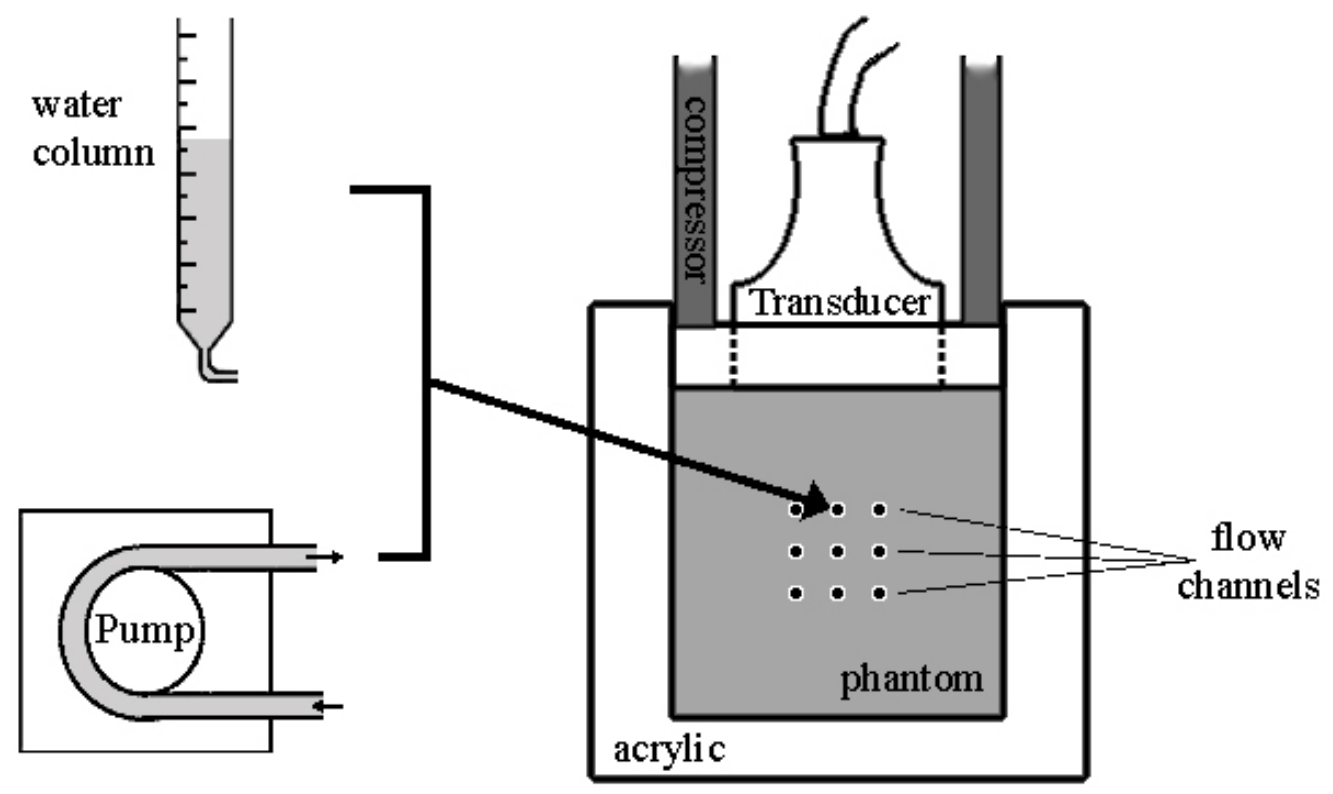

Fig. 1. 


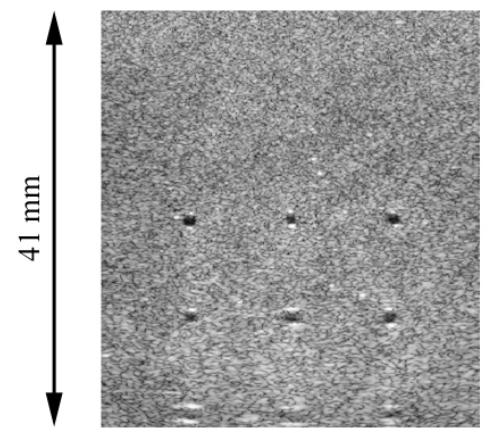

(a)

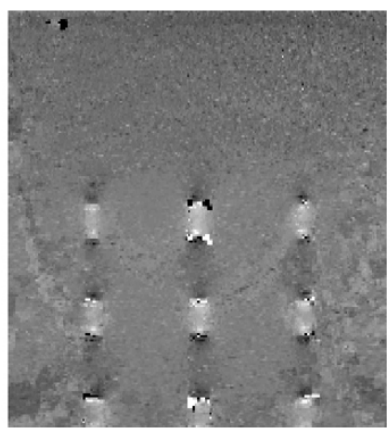

(b)

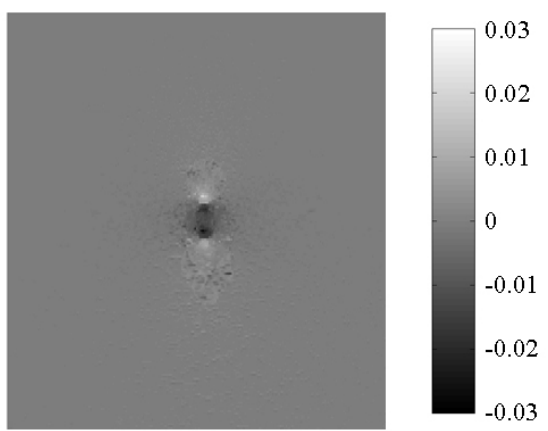

(c)

Fig. 2. 


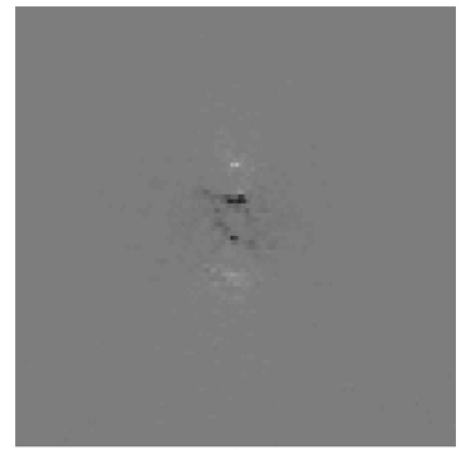

(a)

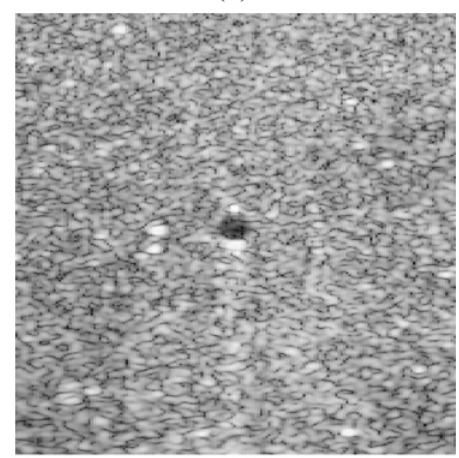

(d)

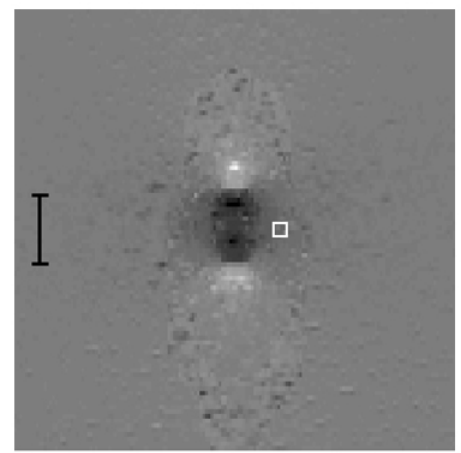

(b)

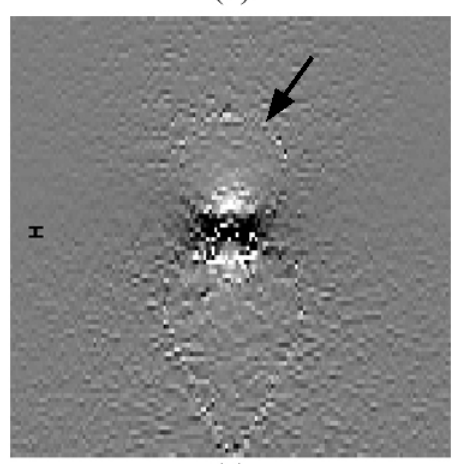

(e)
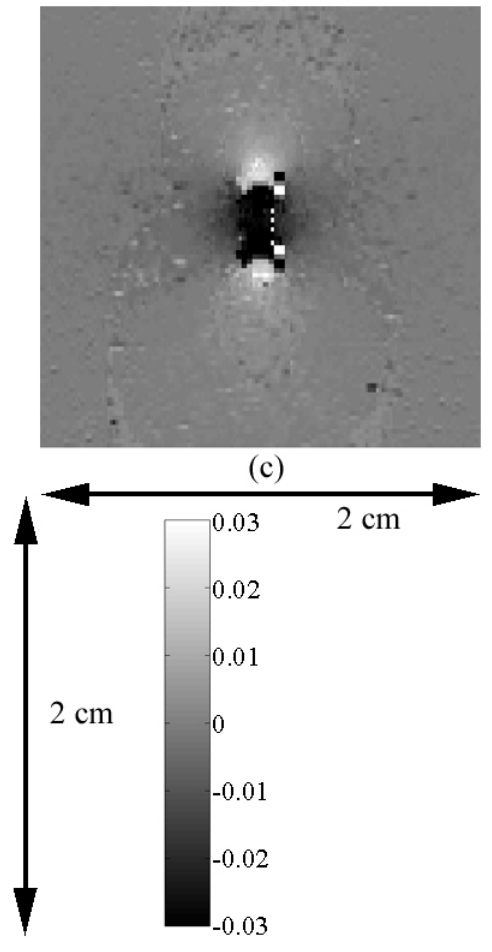

Fig. 3. 


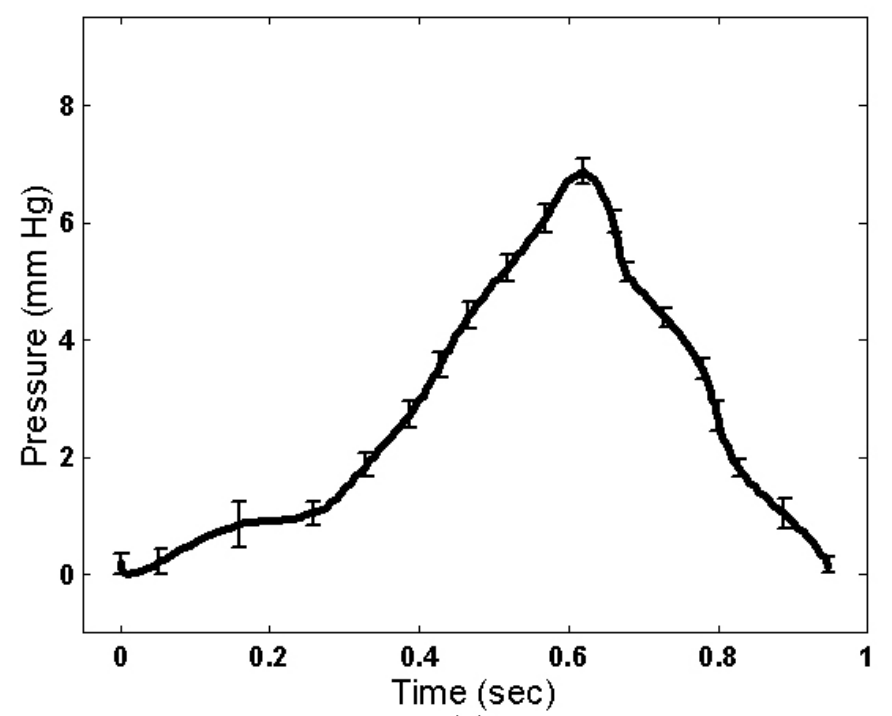

(a)

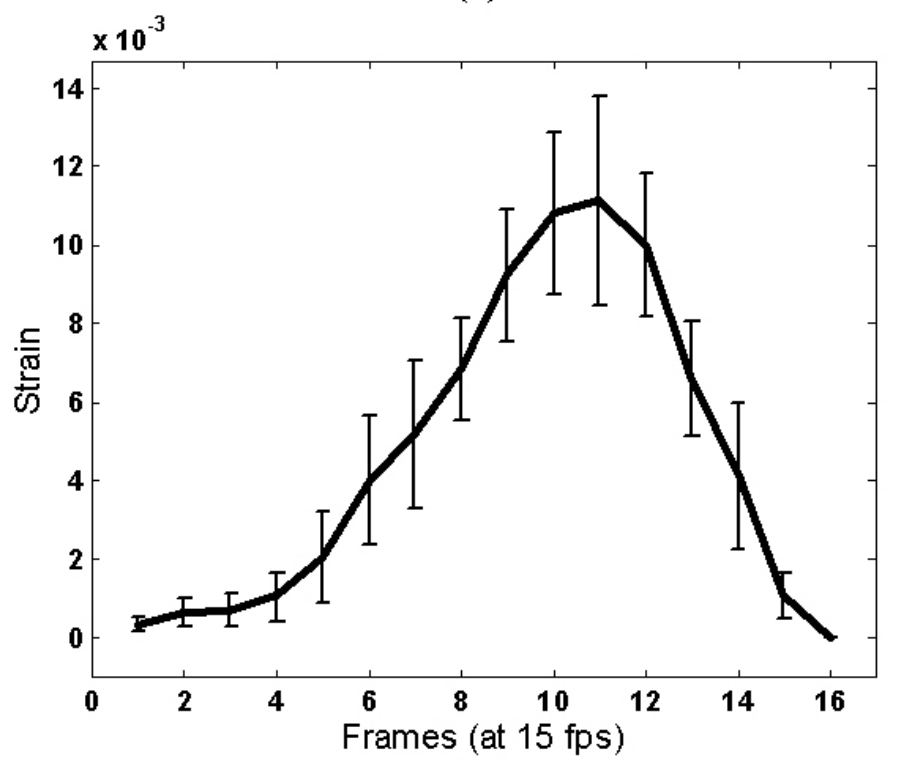

(b)

Fig. 4. 


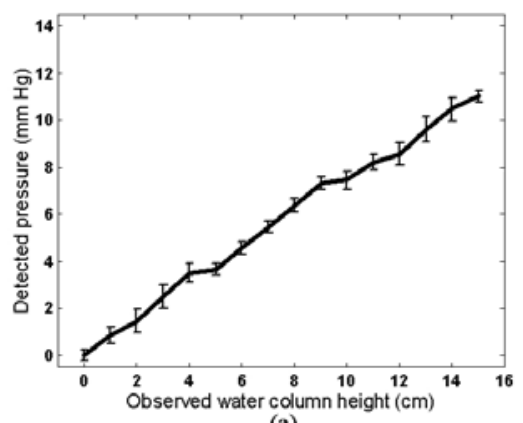

(a)

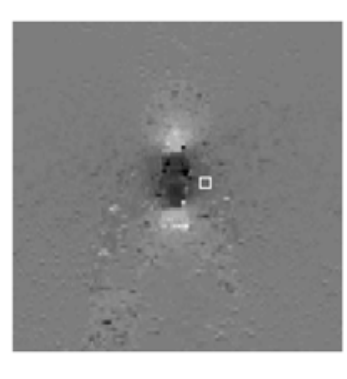

(b)

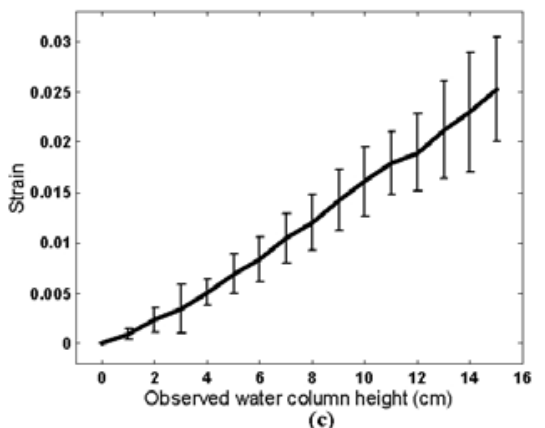

(c)

Fig. 5. 


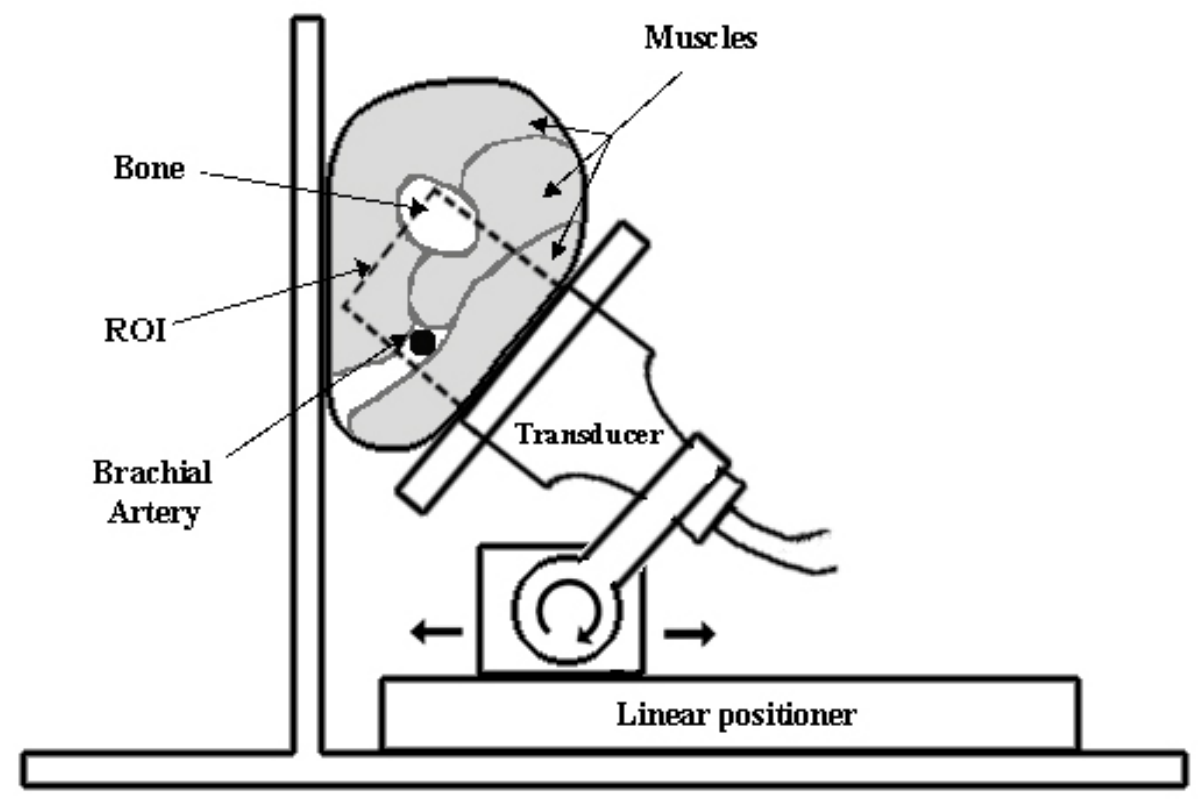

Fig. 6. 


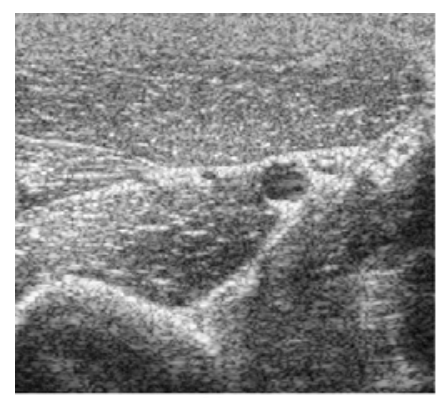

(a)

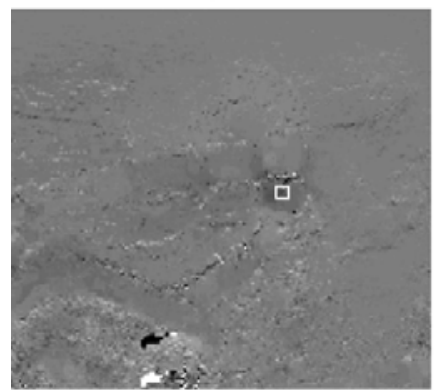

(d)

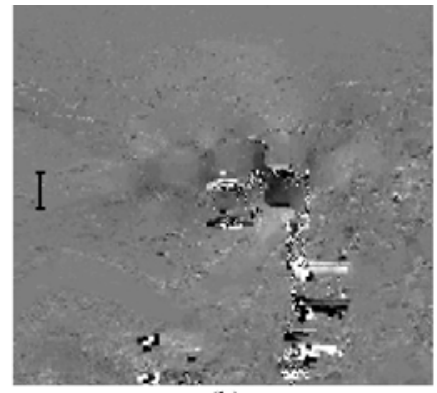

(b)

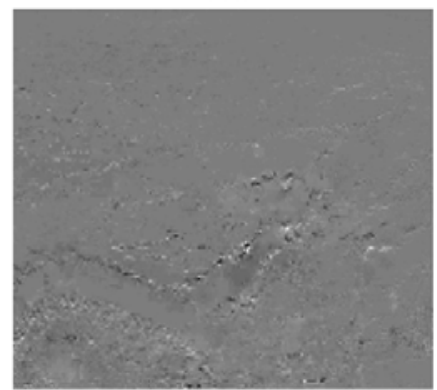

(e)

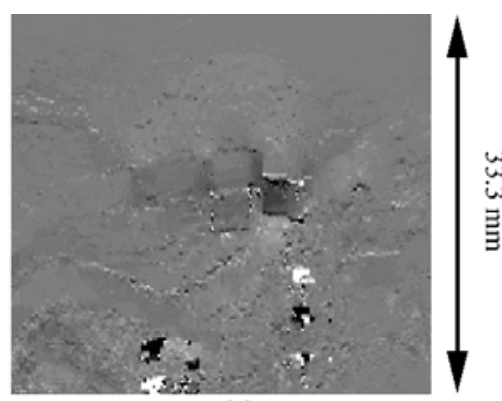

(c)

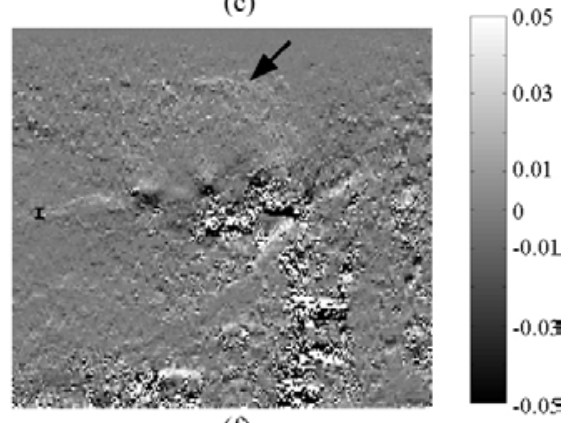

(f)

Fig. 7. 


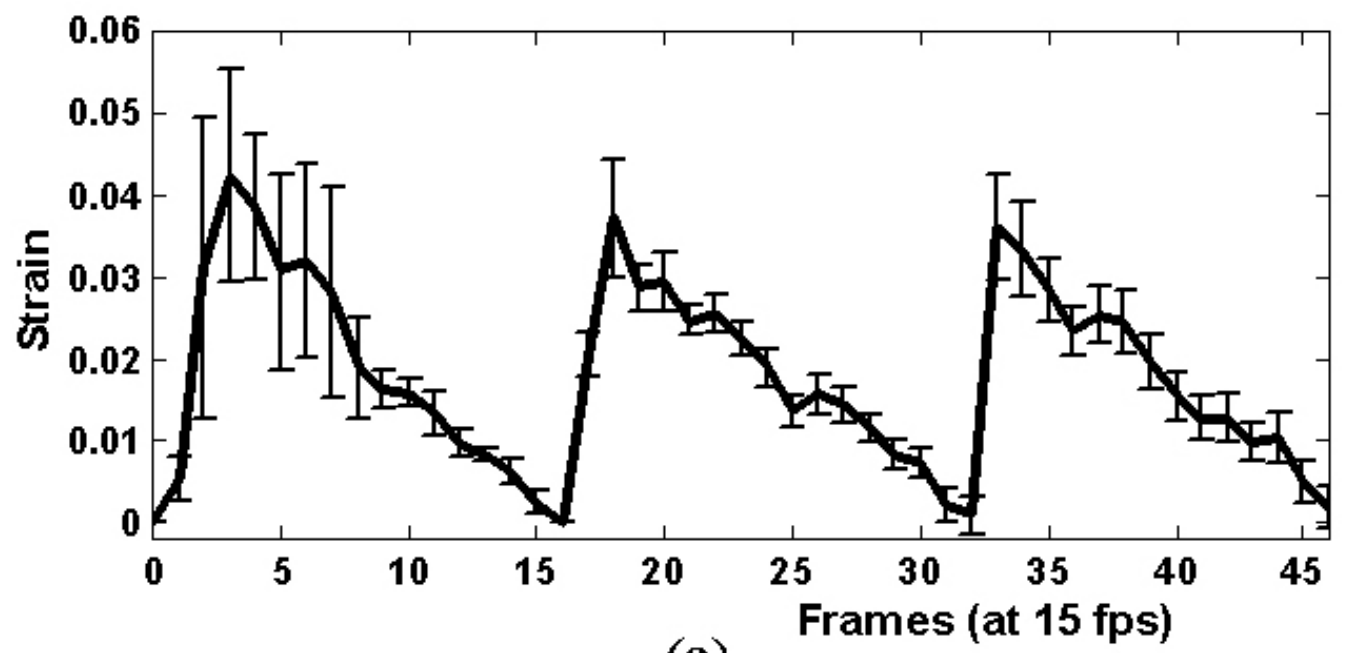

(a)
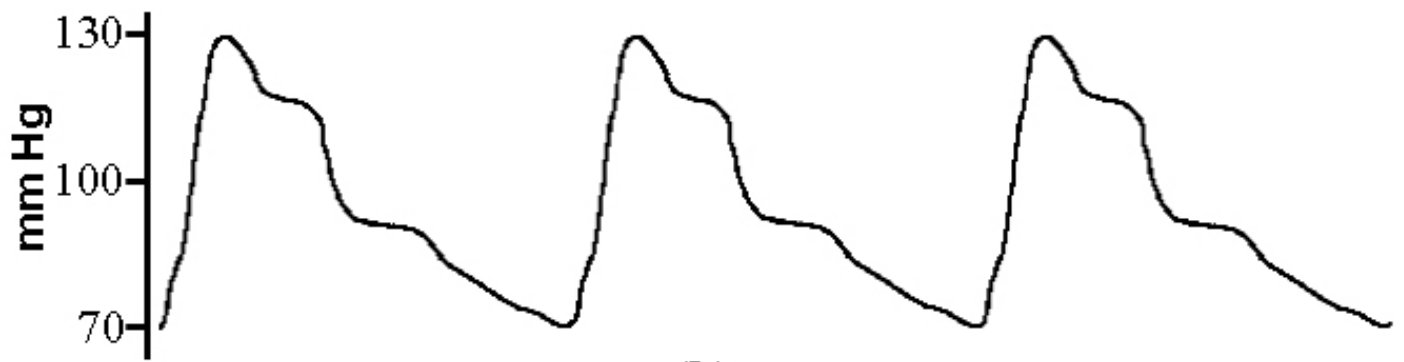

(b)

Fig. 8. 


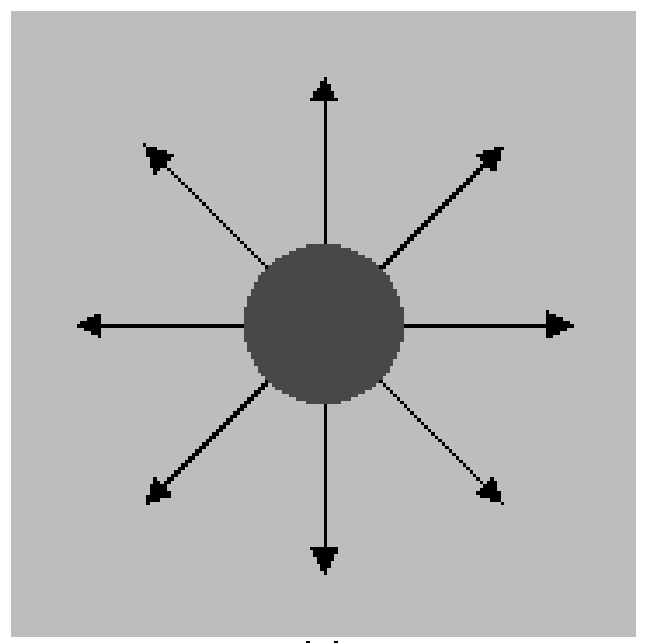

(a)

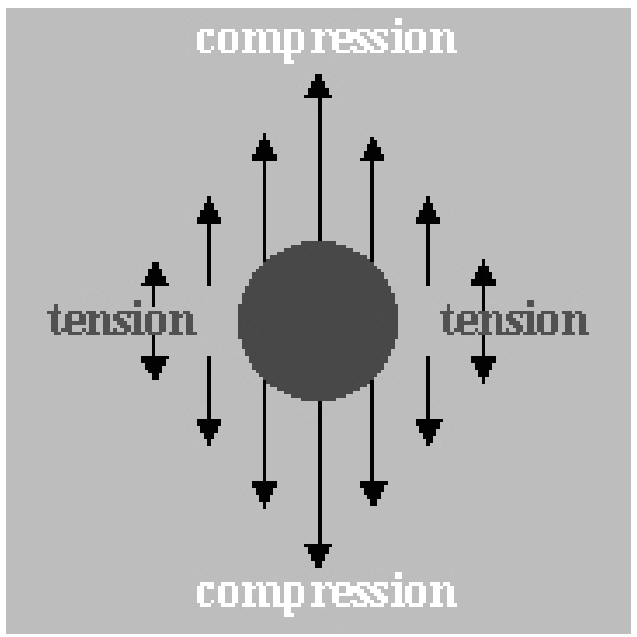

(b)

Fig. 9. 


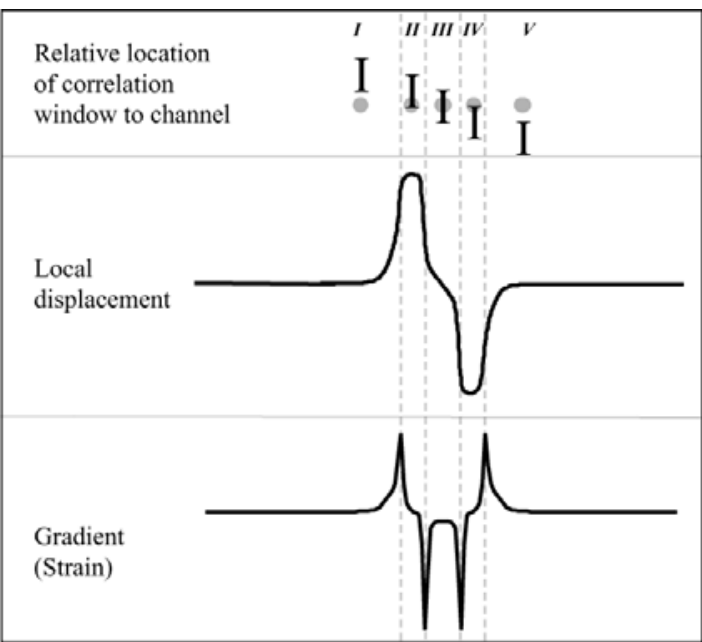

(a)

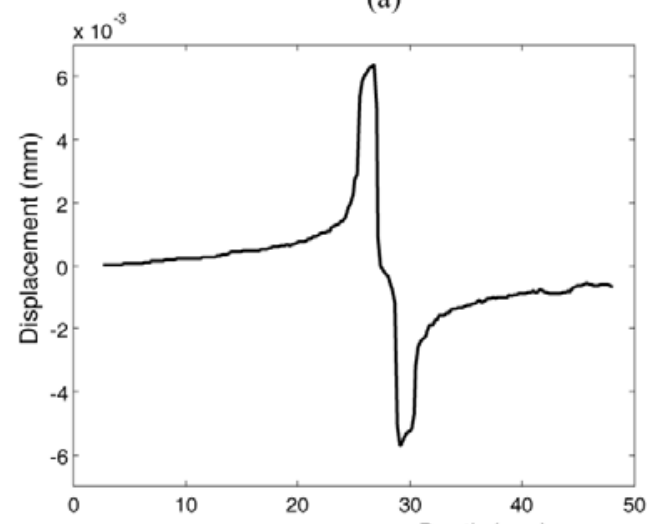

(c) Depth $(\mathrm{mm})$

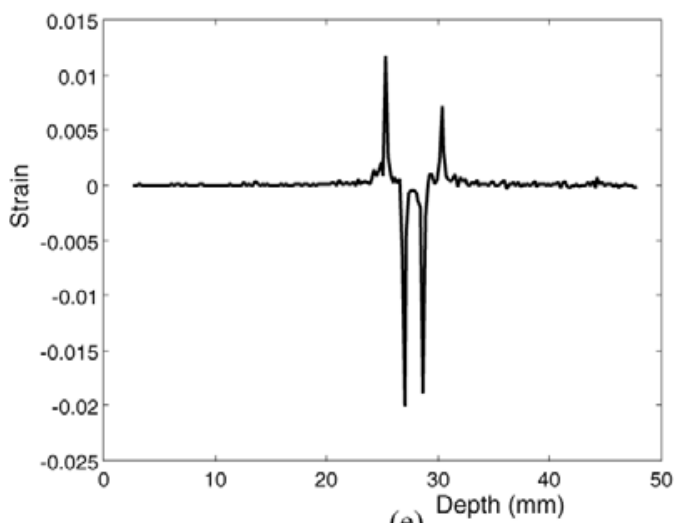

(e)

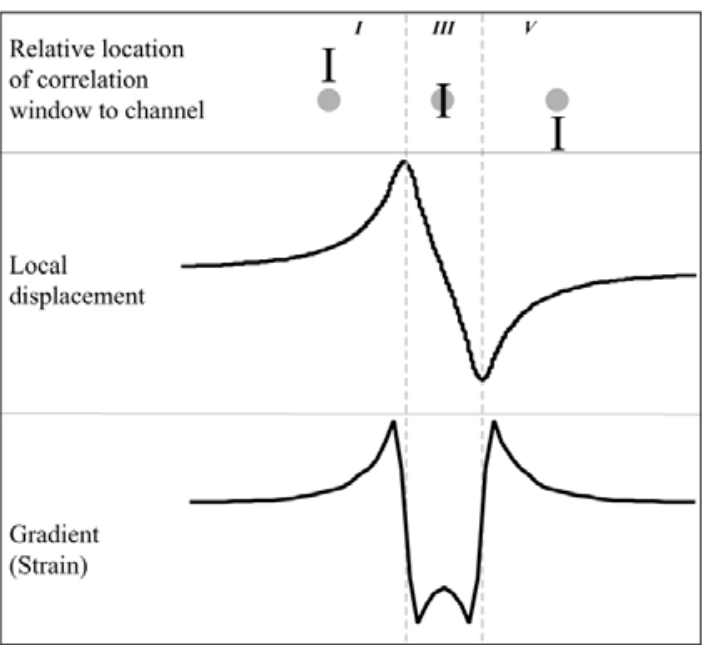

(b)

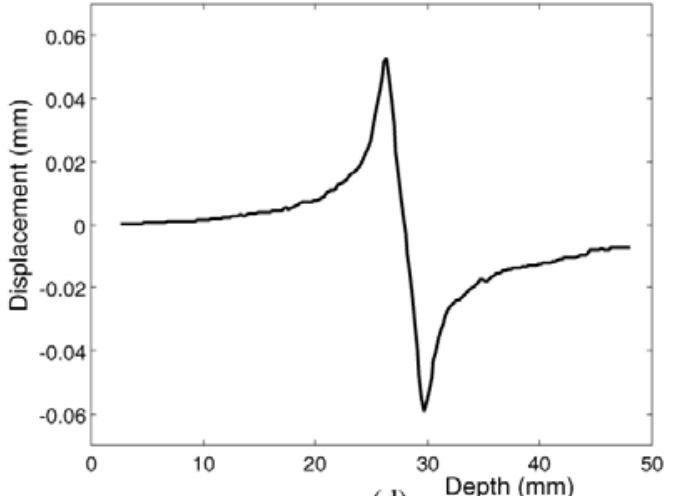

(d)

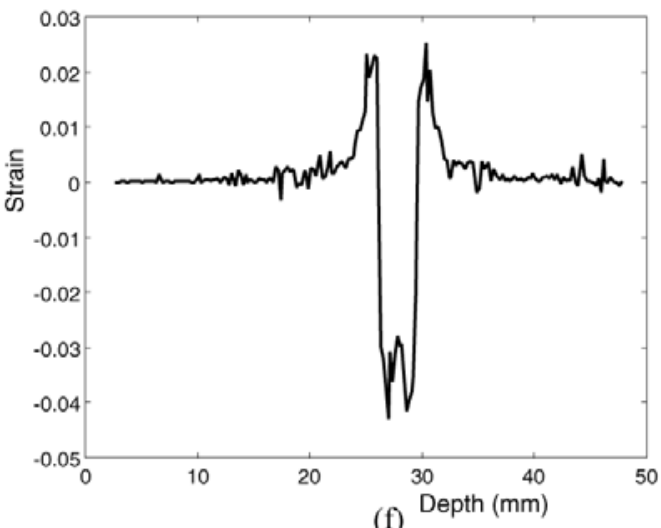


Fig. 10. 


\section{Figure Legends}

Fig. 1. Setup of the elasticity-flow phantom and scanning apparatus. Channel flow is directed into the plane. A water column of adjustable height can be connected to the flow channel while sealing the other end to create internal hydrostatic pressure. A peristaltic pump can be connected to both ends of a channel to create hydrodynamic pressures.

Fig. 2. (a) B-mode image of the elasticity-flow phantom. (b) Strain image of the elasticity-flow in the same region of interest, generated with $2 \%$ external axial compression. (c) A typical strain image of the internal deformation, with only one channel under internal stimulus.

Fig. 3. (a-c) are strain images of a region surrounding a channel under pulsatile flow at different stages of one pulse cycle. They are processed with a correlation window of $3.33 \mathrm{~mm}$, size represented by the "I" in (b). (d) is the same region in B-mode. (e) is the same strain image as (b), but processed with a correlation window of $0.42 \mathrm{~mm}$, represented by the "I" in the image. The arrow in (e) points at the emphasized dipole pattern. The white box in (b) defines the region over which the average strain was estimated for Fig. 4b.

Fig. 4. (a) The pressure profile of one cycle of the pulsatile flow is generated and plotted by averaging several cycles. (b) The average strain magnitude in boxed region in Fig $3 b$ is plotted for the entire pulse cycle. Error bars indicate one standard deviation of the mean. 
Fig. 5. (a) The measured pressure variation inside a channel is plotted against the observed water column height for the hydrostatic pressure experiment. Error bars indicate one standard deviation of the mean over 5000 samples in 5 sec. (b) A typical strain image of the static internal deformation. (c) The average strain magnitude in boxed region in (b) is plotted as a function of water column height, with error bars indicating one standard deviation of the mean.

Fig. 6. Upper right arm rests on the slanted transducer-embedded plate while the echo data of the cross section is acquired. The upper arm, with its cross-sectional anatomy shown in the figure, is positioned horizontally in this view. The positioning device and rotary unit accommodate arms of different sizes.

Fig. 7. (a) is the B-mode image of a region around the brachial artery. (b-e) display the strain images of the brachial artery from peak systole to near end diastole, processed with a $3.33 \mathrm{~mm}$-long correlation window represented by "I" in (b). The white box in (d) defines the region over which the average strain was estimated for Fig. 8a. (f) is the same strain image as (b), but processed with a $0.83 \mathrm{~mm}-10 n g$ correlation window. The arrow in (f) points at an asymmetric dipole pattern from three pulsing vessels.

Fig. 8. (a) The average strain magnitude in the boxed region in Fig 7d is plotted for three cardiac cycles, with error bars illustrating one standard deviation of the mean. (b) The shape of a normal pressure wave at brachial artery is drawn for three cardiac cycles. The resemblance between these plots illustrates a relationship between the strain observed and the pressure variation inside the artery. 
Fig. 9. The dark gray circle represents a fluid flow channel in the phantom, whereas the light gray background is the gelatin. (a) The arrows indicate the direction of forces acted upon the regions around the channel as the channel expands radially. (b) Our strain algorithms sense the axial strain caused by the axial components of the forces shown by the arrows. This results in compression above and below the channel, while tension in regions lateral to the channel.

Fig. 10. Displacement and strain estimations are affected by the extent of channel expansion and the size of the correlation window. The left column (a, c, e) shows the effects when the expansion is small or the correlation window is large; the right column $(b, d, f)$ shows the effects when the expansion is large or the correlation window is small (see text). (a) and (b) are the expected shapes (not drawn to scale) of local displacement and strain profiles along the center axial line at the channel. (c) and (e) are real displacement and strain estimates corresponding to the center axial line in Fig 3a. (d) and (f) are real displacement and strain estimates corresponding to the center axial line in Fig 3c. 